\title{
SOLUÇÃO DA EQUAÇÃO CARACTERÍSTICA NA ANÁLISE DINÂMICA DE UM PÓRTICO PLANO UTILIZANDO O MÉTODO DE NEWTON-RAPHSON
}

\section{SOLUTION OF THE CHARACTERISTIC EQUATION IN THE DYNAMIC ANALYSIS OF A PLANE FRAME USING THE NEWTON-RAPHSON METHOD}

\author{
Gustavo Alberto Aleandri ${ }^{1}$, Adriana Elisabete Limberger ${ }^{2}$, Thiago Augusto Bertuzzo ${ }^{3}$, Gustavo Henrique \\ Dalposso ${ }^{4}$, Rodnny Jesus Mendoza Fakhye ${ }^{5}$
}

Recebido em 03 de novembro de 2012; recebido para revisão em 07 de novembro de 2012; aceito em 12 de novembro de 2012; disponivel on-line em 04 de dezembro de 2012.

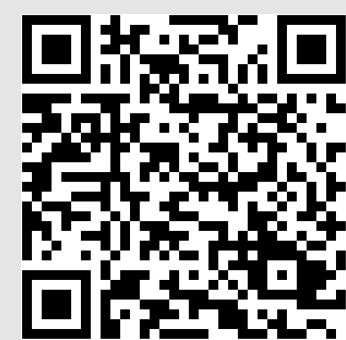

PALAVRAS CHAVES:

Estruturas de concreto armado;

Análise dinâmica de pórticos;

Método de Newton Raphson

\section{KEYWORDS:}

Reiforced Concrete Structures;

Dynamic analysis;

Newton-Raphson

Method.

\begin{abstract}
RESUMO: A análise estrutural em engenharia é de fundamental importância para o projeto correto e desempenho adequado ao longo da vida útil de uma estrutura. Avaliar o comportamento e definir os parâmetros adequados evita, quase sempre, danos futuros e até mesmo diminuem o impacto de desastres como sismos e terremotos, vendavais, entre outros. Acerca disso, o trabalho proposto apresenta o desenvolvimento de um algoritmo auxiliar na determinação de parâmetros contribuintes na análise estrutural, sendo eles as frequências de vibração e os modos de vibração. Para isso, apresenta-se um modelo numérico para a análise dinâmica de um pórtico plano. A solução da equação característica é obtida utilizando o método de Newton-Raphson, programado em linguagem $R^{\circ}$. O sistema a ser analisado é caracterizado por um pórtico plano de dois andares, supondo serem as vigas de rigidez infinita. Determinam-se a matriz de rigidez e a matriz de massa e calculam-se as frequências de vibração, que tem por correspondentes os auto vetores, que são os modos de vibração da estrutura proposta.
\end{abstract}

ABSTRACT: Structural analysis in engineering is crucial for proper design and correct behavior of structures. Correct evaluation of the behavior and set the appropriate parameters avoid, almost always, future damage and even reduce the impact of disasters like seism vibrations and earthquakes, windstorms, and others. From this, the proposed paper presents the development of an algorithm to calculate auxiliary parameters contributing to structural analysis, known as vibration frequencies and mode of shapes. We present a numerical model for dynamic analysis of a plane frame. The solution of the characteristic equation is obtained using the NewtonRaphson method, programmed in $\mathrm{R}^{\circledR}$ language. The system under consideration is characterized by a plane frame with two floors, supposing beams of infinite stiffness. After the calculation of stiffness and mass matrix we obtain the frequencies of vibration, which are associated to the eigenvectors that represent the vibration modes of the proposed structure.

\footnotetext{
* Contato com os autores:

1e-mail : gustavoaleandri@hotmail.com (G. A. Aleandri)

${ }^{2}$ e-mail : adriana.limberger@hotmail.com (A. E. Limberger)

${ }^{3}$ e-mail : thiago_bertuzzo@hotmail.com (T. A. Bertuzzo)

Graduandos em Engenharia Civil da Universidade Tecnológica Federal do Paraná (UTFPR)

${ }^{4}$ e-mail : gustavodalposso@utfpr.edu.br (G. H. Dalposso)

Matemático, Mestre em Engenharia Agrícola e Professor MSc. Universidade Tecnológica Federal do Paraná (UTFPR)

${ }^{5}$ e-mail : rodnny@utfpr.edu.br (R. J. M. Fakhye)

Engenheiro Civil, Mestre em Engenharia Civil e Professor MSc. Universidade Tecnológica Federal do Paraná (UTFPR)
} 


\section{INTRODUÇÃO}

A Engenharia Estrutural é uma área da Engenharia Civil que trata da concepção de projetos, planejamento, construção e manutenção de sistemas estruturais para moradia, trabalho, lazer ou transportes (MAIA e BORGES, 2005) e os materiais geralmente utilizados para cada tipo de empreendimento compreendem o aço, o concreto, a madeira, pedra, fibras e plásticos, aplicados de forma adequada conforme as especificações do projeto. As estruturas são concebidas de maneira a resistir a forças internas e externas, como o seu peso próprio, o peso dos objetos a serem suportados pela estrutura, as condições climáticas, solicitações conferidas durante a sua vida útil e as vibrações.

Face aos problemas de degradação que podem ser observados, durante as últimas décadas verifica-se uma tendência mundial no que diz respeito à elaboração de projetos de engenharia que têm em foco à durabilidade e a extensão da vida útil das estruturas de concreto armado. Desse modo, as vibrações podem ser caracterizadas como um problema que as estruturas possivelmente enfrentarão durante o seu período de vida útil, e conhecê-las e considerá-las nos cálculos estruturais torna-se uma tarefa elementar (POSSAN, 2010).

Além dos fatores citados anteriormente, esses cálculos interferem diretamente na capacidade sustentável das edificações, uma vez que sendo maior seu tempo de vida útil, as reservas de matérias-primas serão poupadas. Com essa preocupação, MEHTA (2001) defende o desenvolvimento da capacidade de se projetar estruturas para 500 anos de vida útil, em vez de 50 . Desta forma multiplica-se por um fator 10 a produtividade das reservas de muitas matérias-primas comumente utilizadas no setor da construção civil em todo o mundo.

As vibrações são caracterizadas pelo movimento oscilatório de um corpo em torno de um ponto fixo e podem ser de caráter longitudinal, flexional ou torcional. São classificadas também de acordo com a amplitude, frequência, comprimento de onda, velocidade e meio de propagação.

As vibrações podem ser ocasionadas, em Engenharia Civil, pelo risco geotécnico, ou seja, o risco onde se verifique probabilidade de danos a edifícios e pessoas devido a problemas na interface estruturaterreno (ou estrutura-maciço rochoso). O maciço pode criar problemas na construção de obras por vários motivos, como o inadequado planejamento ou realização dos ensaios de campo, juntamente com a diferença da geometria ou das propriedades do maciço, relativamente ao previsto no projeto, água subterrânea, propriedades adversas, etc. (LONGO e GAMA, 2007).

Outro fator que acarreta movimentos oscilatórios nas estruturas são os sismos. Um sismo constitui a liberação de forma repentina de energia em falhas de blocos rochosos ou nas fronteiras das placas tectônicas. Essa liberação é responsável pela geração e propagação de diversos tipos de oscilações no solo, que se atingem uma estrutura de concreto, por exemplo, originam comprometimentos, como fissuras, e em casos extremos, o rompimento.

Fatores climáticos também podem causar diversos tipos de vibrações sendo o fator mais comum o vento. Os efeitos do vento são de caráter dinâmico, entretanto na maioria das construções esses efeitos podem ser substituídos por ações estáticas equivalentes. Em edificações esbeltas e flexíveis, principalmente aquelas com baixas frequências naturais de vibração $(f<$ $1.0 \mathrm{~Hz}$ ), os efeitos dinâmicos devem ser considerados (CHAMBERLAIN apud HAUCH, 2010).

Causadores específicos também podem ser citados quando se tratam de vibrações, como o uso de explosivo em escavações, utilização de equipamentos pesados (cravadores de estacas e perfuradores), veículos de trânsito urbano quotidiano, etc.

A análise desses esforços como os sismos e a ação dos ventos pode ser realizada através da obtenção e resolução da equação característica do sistema estrutural. Esse sistema pode ser caracterizado, quando para edifícios, em pórticos. Toda estrutura formada por barras vinculadas entre si é denominada pórtico espacial. $\mathrm{Na}$ prática, é possível isolar subconjuntos do pórtico espacial e analisá-los como estruturas independentes, ligadas umas às outras por vínculos, transformando-as em pórticos planos. O pórtico plano é uma estrutura formada por barras coplanares e submetida a cargas também pertencentes a este plano.

Após a obtenção da equação característica através das matrizes características (rigidez e massa) e da frequência, é possível determinar com escolhida precisão os modos de vibração da estrutura. Neste trabalho, é utilizado o método de aproximação de zeros reais para funções reais, definido por Newton-Raphson. Conhecendo os modos de vibração, é possível prever o comportamento da estrutura ao receber diferentes tipos de esforços, podendo assim prevenir inadequações estruturais.

\section{OBJETIVOS}

O presente trabalho tem por objetivo propor um algoritmo em linguagem $R^{\oplus}$ para estimar os zeros 
reais de funções reais na solução da equação característica na análise do comportamento dinâmico de um pórtico plano quando submetido à ação de vibrações mecânicas.

\section{MATERIAIS E MÉTODOS}

\subsection{DINÂMICA DE ESTRUTURAS}

A dinâmica diz respeito ao movimento e suas causas. Sendo assim, dinâmica de estruturas é a parte que estuda o que as ações da natureza causam na estrutura e como ela reage. A análise consiste em quantificar os movimentos, velocidades, acelerações, esforços e tensões que ocorrem na estrutura, variando com o tempo. (BEER et al., 2010). Três tipos de força são os mais decorrentes nas estruturas: a força restauradora, a força dissipativa e a força de inércia.

A força restauradora, que é a força que age sobre a estrutura de forma a recuperar o seu formato original, apresenta-se diretamente proporcional ao seu deslocamento. Essa força é descrita pela Lei de Hooke, definida pela Equação 1:

$$
F=k \cdot x
$$

Em que o esforço produzido é diretamente proporcional à distância percorrida e pela rigidez da mola. A força de dissipação é observada quando ocorre a perda de energia pelo atrito interno da estrutura (atrito entre barras, barras e parafusos). Já a força de inércia é a força que o corpo tem de resistir ao movimento e é baseada na segunda Lei de Newton, conforme a Equação 2:

$$
F=m \cdot a
$$

Na qual a força produzida sobre um corpo de massa " $\mathrm{m}$ " é diretamente proporcional à sua aceleração a (BEER et al., 2006). Alguns fatores que levam a estrutura a ter movimentações são: sismos, ventos, movimentação de pessoas (automóveis) e equipamentos de construções e industriais (britadeiras, moinhos).

De acordo com CLOUGH e PENZIEN (1995), praticamente qualquer tipo de sistema estrutural pode ser submetido a cargas dinâmicas determinísticas e não determinísticas durante o seu tempo de vida. As cargas dinâmicas determinísticas podem ser caracterizadas pela condução direta de deslocamentos ao longo do tempo a partir de um carregamento conhecido e variável no tempo, por outro lado, se a variação de tempo não é completamente conhecida, mas pode ser definida no sentido estatístico, o carregamento é determinado dinâmico não determinístico, ou aleatório.
Do ponto de vista analítico, é conveniente dividir cargas determinísticas em duas categorias básicas: periódicas e não periódicas. Algumas formas típicas de cargas prescritas e exemplos de situações na qual tais cargas podem ser desenvolvidas são mostradas na Figura 1.

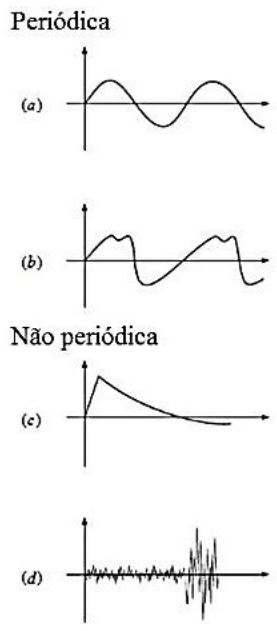

Histórico de cargas
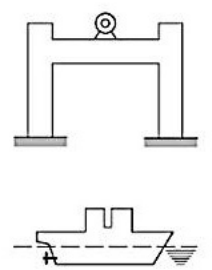

Rotação da hélice da popa de navios

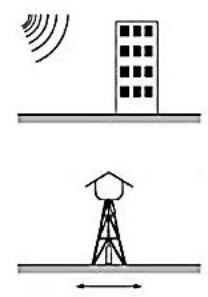

Exemplos típicos

Máquina rotativa desbalanceada de construções

Pressão exercida pela explosão de bombas em edificios

Efeito de terremotos em caixas d'água
Figura 1: Características e origens de cargas dinâmicas típicas: (a) harmônico simples; (b) complexo; (c) impulsivo; (d) longa duração. Fonte: CLOUGH e PENZIEN (1995).

\subsection{VIBRAÇÕES LIVRES NÃO-AMORTECIDAS}

Um carregamento dinâmico consiste em qualquer tipo de carregamento cuja magnitude, direção e/ou posição varia no tempo. Em geral, a resposta estrutural a qualquer carregamento dinâmico é expressa basicamente em termos dos deslocamentos da estrutura (CLOUGH e PENZIEN, 1995). No caso estudado nesse trabalho, o carregamento aplicado é de caráter dinâmico, pois os deslocamentos da estrutura dependem não só do esforço estático, mas também das forças inerciais. Para um sistema sem amortecimento, a equação governativa do movimento em vibração livre de sistemas lineares com múltiplos graus de liberdade é definida através da Equação 3:

$$
\underline{M} \ddot{u}+\underline{K u}=0
$$

Em que $M$ é a matriz de massa, $K$ é a matriz de rigidez, $\ddot{u}$ é a aceleração do movimento e $u$ é o deslocamento. $\mathrm{O}$ deslocamento, e consequentemente a aceleração, são dependentes do tempo. A equação de vibração livre, portanto, para um sistema não amortecido num dos modos de vibração naturais é dada pela Equação 4:

$$
u(t)=q_{n}(t) \underline{\phi_{n}}
$$


Onde $\phi_{n}$ é o modo de vibração natural $n$ invariante no tempo e $q_{n}(t)$ é a variação dos deslocamentos com o tempo. Definindo a variação com a Equação 5:

$$
q_{n}(t)=A_{n} \cos \left(\omega_{n} t\right)+B_{n} \sin \left(\omega_{n} t\right)
$$

Substituindo na equação governativa obtémse a Equação 6:

$$
u(t)=\underline{\phi_{n}}\left[A_{n} \cos \left(\omega_{n} t\right)+B_{n} \sin \left(\omega_{n} t\right)\right] \quad \text { Eq.[06] }
$$

Visivelmente, $\phi_{n}$ apresenta-se como solução trivial. Daí exprime-se que o problema de valores e vetores próprios é definido utilizando a Equação 7:

$$
\underline{K \phi_{n}}=\omega_{n}^{2} \underline{M \phi_{n}}
$$

Por outro lado, as soluções não triviais só poderão ser obtidas obedecendo a Equação 8:

$$
\operatorname{det}\left(\underline{K}-\omega^{2} \underline{M}\right)=0
$$

Finalmente, expandindo-se o determinante, obtém-se um polinômio de ordem $n$ e $\omega_{n}^{2}$, sendo $\omega_{n}$ definido por frequência natural de vibração. A equação, determinada equação característica, possui $n$ raízes reais positivas, pois as matrizes de massa e rigidez são simétricas e positivas definidas. Quando a equação é conhecida, como no caso estudado nesse trabalho, é possível determinar o vetor correspondente modo de vibração natural $\underline{\phi_{n}}$.

\subsection{O MÉTODO DE NEWTON-RAPHSON}

De acordo com RUGGERIO e LOPES (2008), seja $f(x)$ uma função contínua em $[a, b]$, o intervalo I que contém uma raiz $\alpha$ da equação $f(x)=0$. O método de Newton-Raphson consiste em transformar essa equação em uma equação equivalente $x=\varphi(x)$ a partir de uma aproximação inicial $x_{0}$ e gerar uma sequência $\left\{x_{k}\right\}$ de aproximações para $\alpha$ pela função de iteração (Equação 9):

$$
\varphi\left(x_{k}\right)=x_{k+1}
$$

O que o método de Newton faz, na tentativa de garantir e acelerar a convergência, é escolher para função de iteração a função $\varphi(x)$ tal que $\varphi(\alpha)=0$ utilizando o coeficiente angular da reta que passa pela aproximação inicial. Conforme apresentando pelo conjunto de Equações 10 a 14

$$
\begin{gathered}
y=f\left(x_{0}\right)+f^{\prime}\left(x_{0}\right) x-f^{\prime}\left(x_{0}\right) x_{0} \\
\text { Se } y=0 \text {, então: } \\
f\left(x_{0}\right)+f^{\prime}\left(x_{0}\right) x-f^{\prime}\left(x_{0}\right) x_{0}=0 \\
f^{\prime}\left(x_{0}\right) x=f^{\prime}\left(x_{0}\right) x_{0}-f\left(x_{0}\right) \\
x=\frac{f^{\prime}\left(x_{0}\right) x_{0}}{f^{\prime}\left(x_{0}\right)}-\frac{f\left(x_{0}\right)}{f^{\prime}\left(x_{0}\right)} \\
x=x_{0}-\frac{f\left(x_{0}\right)}{f^{\prime}\left(x_{0}\right)} .
\end{gathered}
$$

Nessas condições, a convergência do método é válida se, e somente se $f(x), f^{\prime}(x)$ e $f^{\prime \prime}(x)$ forem contínuas em I . Então $\exists \overline{\mathrm{I}} \subset \mathrm{I}$, contendo $\alpha$ tal que se $x_{0} \in \overline{\mathrm{I}},\left\{x_{k}\right\} \rightarrow \alpha$ (CHAPRA e CANALE, 2006).

Utilizando-se recursos computacionais, pôdese programar essa metodologia em linguagem $R^{\oplus}$ para estimar os zeros reais de funções reais (TORGO, 2006).

\subsection{ALGORITMO PARA DETERMINAR ZEROS REAIS DE UMA FUNÇÃO REAL PELO MÉTODO DE NEWTON- RAPHSON}

O algoritmo apresentado no Quadro 1 consiste em definir uma função $f(x)$ qualquer e plotar seu gráfico a fim de determinar a aproximação inicial $x_{0}$ da raiz da equação. Na etapa seguinte, define-se a derivada $f^{\prime}(x)$ e realizam-se as iterações. Enquanto a imagem da aproximação $x$ for maior que o erro $z$, a função toma uma nova aproximação, que foi calculada anteriormente, e os critérios de parada continuam sendo testados. Atingido esse critério, são mostrados em tela a aproximação, o número de iterações realizadas e o valor da função na aproximação.

Quadro 1: Algoritmo utilizado para estimar os zeros reais de funções reais

\#1. Definindo a função

$\mathrm{f}<-$ function $(\mathrm{x})\{\mathrm{f}(\mathrm{x})\}$

\#1.1 Exibindo o gráfico da função para

determinar a aproximação inicial

plot.new()

plot(f,15,50,col="blue")

abline ( $v=0$, col="black")

abline( $\mathrm{h}=0, \mathrm{col}=$ "black")

\#2. Definindo a derivada da função

g<-function $(x)\left\{f^{\prime}(x)\right\}$

\#3. Algoritmo

newton<-function $(x, z)\{$ 
Quadro 1: Algoritmo utilizado para estimar os zeros reais de funções reais (continuação).

iteração<-0

aproximação<-x

while(abs(f(aproximação))>z)\{

nova_aproximação<-aproximação-

$((f($ aproximação $)) /(g($ aproximação $)))$

iteração<-iteração+1

aproximação<-nova_aproximação

\}

print("Aproximação")

print(aproximação,digits=20)

print("Número de iterações")

print(iteração)

print("Valor da função na aproximação")

print(f(aproximação), digits=20)

\}

\#Teste com aproximação inicial $x$ e erro $z$ newton $(x, z)$

\section{RESULTADOS E DISCUSSÃO}

\subsection{ANÁLISE DINÂMICA DE UM PÓRTICO PLANO}

Para a análise dinâmica proposta pelo presente trabalho, considera-se um pórtico plano de dois andares, como apresentado na Figura 2, de vigas supostas de rigidez infinita, com módulo de elasticidade $E$ igual à $2.10^{7} \mathrm{kN} / \mathrm{m}^{2}$. Esta aplicação é adaptada de DELGADO e ARÊDE (2000).

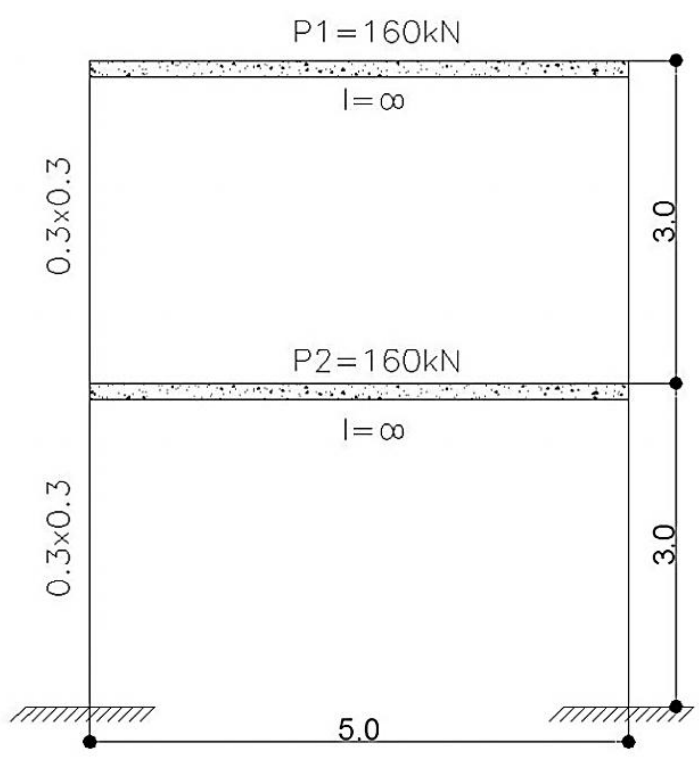

Figura 2: Pórtico plano utilizado para o estudo.

\subsubsection{Determinação da matriz de rigidez}

Para a obtenção da equação característica, deve-se primeiramente determinar as matrizes de rigidez e massa da estrutura a ser analisada. A matriz de rigidez é uma matriz quadrada, de ordem igual à quantidade de pavimentos da estrutura, definida por meio das Equações de 15 a 19. Para sua determinação, devem-se considerar deslocamentos unitários em um pavimento e fixar o outro (Figuras 3 e 4).

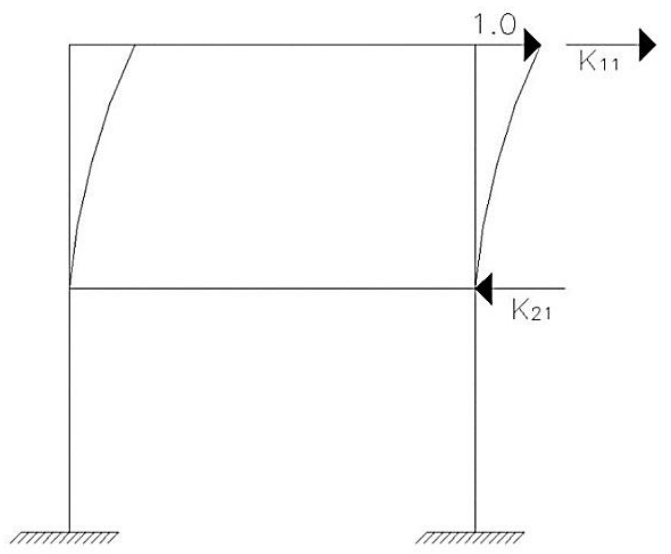

Figura 3: Primeira etapa para a obtenção dos elementos da matriz de rigidez do pórtico plano.

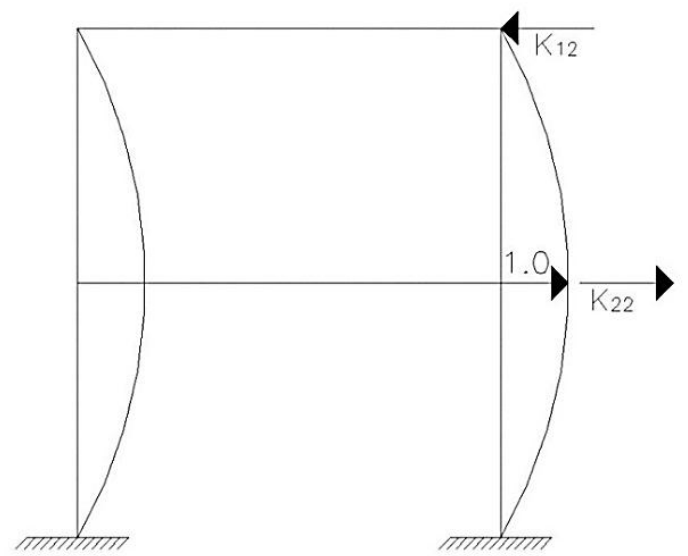

Figura 4: Segunda etapa para a obtenção dos elementos da matriz de rigidez do pórtico plano.

$$
\begin{gathered}
K_{11}=\frac{12 E I}{l^{3}} \times 2=12000 \mathrm{KN} / \mathrm{m} \\
K_{21}=-12000 \mathrm{KN} / \mathrm{m} \\
K_{12}=-12000 \mathrm{KN} / \mathrm{m} \\
K_{22}=24000 \mathrm{KN} / \mathrm{m} \\
\underline{K}=\left[\begin{array}{cc}
12000 & -12000 \\
-12000 & 24000
\end{array}\right]
\end{gathered}
$$

\subsubsection{Determinação da matriz de massa}

A matriz de massa consiste em uma matriz diagonal, de ordem igual à quantidade de pavimentos da estrutura, em que as massas de cada pavimento são 
inseridas na diagonal principal, definida por meio das Equações 20 e 21.

$$
\begin{aligned}
& m_{i}=\frac{160}{9.8}=16,33 \text { ton } \\
& \underline{M}=\left[\begin{array}{cc}
16.33 & 0 \\
0 & 16.33
\end{array}\right]
\end{aligned}
$$

A equação característica pode ser obtida, então, pela relação $\underline{K}-\omega^{2} \underline{M}$, conforme pode-se observar nas Equações 22, 23 e 24.

$$
\begin{gathered}
\underline{K}-\omega^{2} \underline{M}=\left[\begin{array}{cc}
12000-16.33 \omega^{2} & -12000 \\
-12000 & 24000-16.33 \omega^{2}
\end{array}\right] \\
\operatorname{det}\left(\underline{K}-\omega^{2} \underline{M}\right)=0
\end{gathered}
$$$$
\left(12000-16.33 \omega^{2}\right)\left(24000-16.33 \omega^{2}\right)-12000^{2}=0
$$

\subsubsection{Determinação das frequências de vibração}

As velocidades angulares e as frequências de vibração podem ser determinadas por meio das Equações 25 e 26.

Como a equação tem domínio pertencente aos Reais para qualquer intervalo, assim como sua primeira e segunda derivadas, o algoritmo de NewtonRaphson pode ser utilizado. Dessa forma, a partir do algoritmo apresentado no Quadro 1 chegou-se aos seguintes valores para $\omega_{1}$ e $\omega_{2}$ :

$$
\begin{array}{ll}
\omega_{1}=16.76 \mathrm{rad} / \mathrm{s} ; f_{1}=2.7 \mathrm{~Hz} & \text { Eq.[25] } \\
\omega_{2}=43.86 \mathrm{rad} / \mathrm{s} ; f_{2}=7.0 \mathrm{~Hz} & \text { Eq.[26] }
\end{array}
$$

A Figura 5 apresenta o gráfico da função característica e os resultados obtidos pelo método de Newton-Raphson implementado no algoritmo.

Para cada $\omega$ (auto valor) existe um $\phi$ (auto vetor) que caracteriza a forma deformada da estrutura, definindo o modo de vibração do sistema. Como as frequências de vibração $\omega_{1}$ e $\omega_{2}$ são conhecidas, a equação característica pode ser resolvida para os correspondentes vetores de modo de vibração $\underline{\phi_{1}}$ e $\underline{\phi_{2}}$. A metodologia empregada para a determinação de $\underline{\phi_{1}}$ e $\underline{\phi_{2}}$ pode ser observada nas Equações de 27 a 32 .

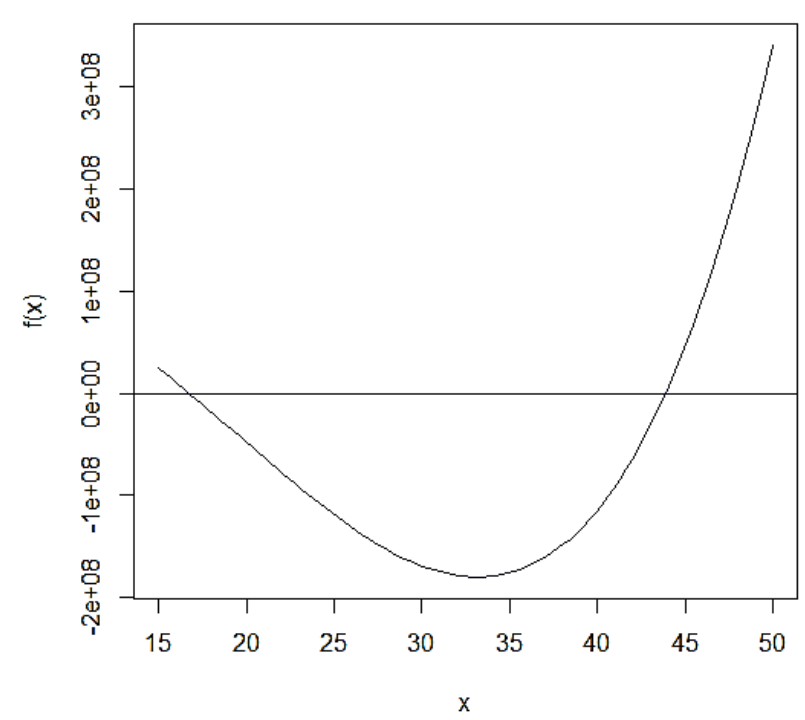

\footnotetext{
$>$ \#Teste com chute inicial 20 e erro 0.005

Figura 5: Gráfico da função da equação característica e resultados obtidos a partir do método de Newton-Raphson para estimar os zeros reais da função.

\subsubsection{Determinação dos modos de vibração}

A determinação do primeiro modo de vibração, conforme apresentado na Figura 6 , pode ser feita por meio das Equações 27, 28 e 29.

$$
\left(\underline{K}-\omega_{1}^{2} \underline{M}\right) \cdot \underline{\phi_{1}}=0
$$

$$
\left[\begin{array}{cc}
12000-16.33 \times(16.76)^{2} & -12000 \\
-12000 & 24000-16.33 \times(16.76)^{2}
\end{array}\right]\left\{\begin{array}{l}
\phi_{11} \\
\phi_{21}
\end{array}\right\}=0
$$

Fazendo $\phi_{11}=1.0 \rightarrow \phi_{21}=0.618$ tem-se:

$$
\underline{\phi_{1}}=\left\{\begin{array}{c}
1.0 \\
0.618
\end{array}\right\}
$$




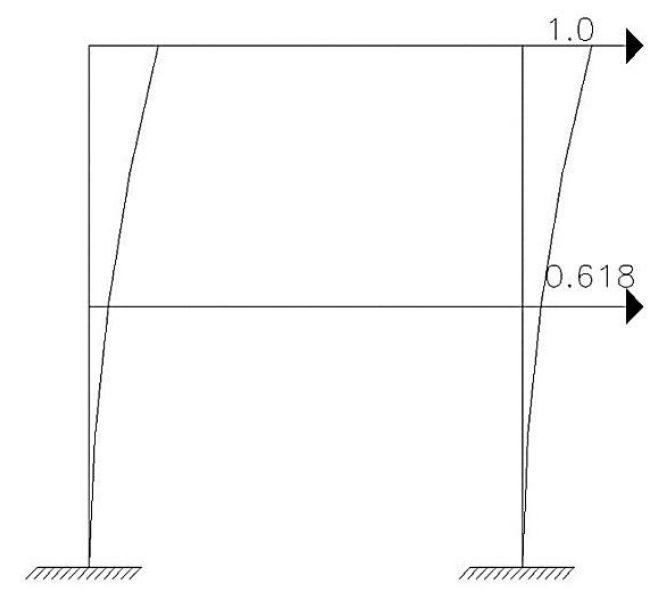

Figura 6: Primeiro modo de vibração do pórtico plano.

A determinação do segundo modo de vibração, conforme apresentado na Figura 7, pode ser feita por meio das Equações 30, 31 e 32.

$$
\begin{array}{cc}
\left(\underline{K}-\omega_{2}^{2} \underline{M}\right) \cdot \underline{\phi_{2}}=0 & \text { Eq.[30] } \\
{\left[\begin{array}{cc}
12000-16.33 \times(43.86)^{2} & -12000 \\
-12000 & 24000-16.33 \times(43.86)^{2}
\end{array}\right]\left\{\begin{array}{l}
\phi_{11} \\
\phi_{21}
\end{array}\right\}=0}
\end{array}
$$

Fazendo $\phi_{22}=1.0 \rightarrow \phi_{12}=-0.618$ tem-se:

$$
\underline{\phi_{1}}=\left\{\begin{array}{c}
-0.618 \\
1.0
\end{array}\right\}
$$

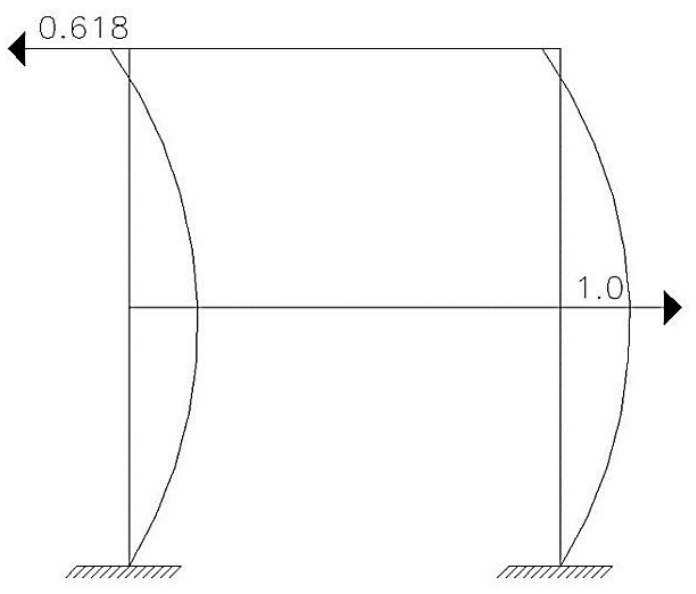

Figura 7: Segundo modo de vibração do pórtico plano.

\section{CONCLUSÃO}

A partir das análises realizadas, pode-se inferir que os ferramentais computacionais apresentam-se como uma boa alternativa na resolução de problemas relativos à Engenharia Estrutural. O problema proposto parece ser relativamente simples quando analisado com somente dois graus de liberdade, entretanto quando a avaliação estrutural passa a ser de mais de três pavimentos, as iterações matemáticas tornam-se mais onerosas. O algoritmo apresenta-se, portanto, viável para a resolução de problemas com as características anteriormente citadas. O método desenvolvido assegura precisão numérica bastante apurada, o que confere maior precisão nos resultados obtidos, garantindo maior confiabilidade à análise dos dados.

\section{REFERÊNCIAS BIBLIOGRÁFICAS}

BEER, F. P.; JOHNSTON Jr., E. R.; DEWOLF, J. T. Resistência dos Materiais - Mecânica dos Materiais. 4. ed. Rio de Janeiro: McGraw-Hill Interamericana do Brasil. 2010. 758 p.

BEER, F. P.; JOHNSTON Jr., E. R.; EISENBERG, E. R. Mecânica Vetorial para Engenheiros - Dinâmica. 7.ed. São Paulo: McGraw-Hill Interamericana do Brasil. 2006. 621 p.

CHAPRA, S. C.; CANALE, R. P. Numerical Methods for Engineers. 5. ed. New York: McGraw-Hill. 2006. 926 p.

CLOUGH, R.W.; PENZIEN, J. Dynamics of Structures. 3. ed. New York: McGraw-Hill. 1995. 752 p.

DELGADO, R.; ARÊDE, A. Análise dinâmica de sistemas com n graus de liberdade. 2000. Disponível em: < http://civil.fe.up.pt/pub/apoio/ano5/de/acetatos/capit ulo_9_10.pdf>. Acessado em 1 nov. 2012.

HAUCH, A. S. Análise da Estabilidade Global de Estruturas de Concreto Armado. 2010, 75 p. Trabalho de conclusão de Curso (Graduação em Engenharia Civil) - Universidade Regional do Noroeste do Estado do Rio Grande do Sul, Ijuí, 2010.

LONGO, S.; GAMA, C. D. Análise dos Riscos Inerentes às Vibrações Transmitidas às Estruturas. 2007. Disponível em: <http://cegeo.ist.utl.pt/html/investiga/vibra.pdf>. Acessado em: 1 nov. 2012.

MAIA, E. V.; BORGES, O. Uma Análise de Desempenho dos Alunos de Engenharia Civil e Mecânica da UFMG na Aplicação dos Conceitos Básicos na Área de Estruturas. 2005. Disponível em: <http://www.nutes.ufrj.br /abrapec/venpec/conteudo/artigos/1/doc/p751.doc>. Acessado em: 1 nov. 2012.

MEHTA, P. K. Reducing the Environmental Impact of Concrete. Concrete International, v.23, n.10, 2001, 61-66 p.

POSSAN, E. Modelagem da Carbonatação e Previsão de Vida Útil de Estruturas de Concreto em Ambiente Urbano. 2010, 265p. Tese (Doutorado) - Universidade Federal do Rio Grande do Sul, Porto Alegre, 2010. 
RUGGIERO, M. A. G.; LOPES, V. L. R. Cálculo Numérico: Aspectos Teóricos e Computacionais. 2a. ed. São Paulo: Editora Pearson. 2008. 406 p.

TORGO, L. Introdução à Programação em R. 2006. Disponível em: <http://pt.scribd.com/doc/92389615/

Introducao-a-Programacao-em-R>. Acessado em: 1 de novembro de 2012. 